\title{
ANTIPARA (Analysis of Tiny Particles in Aquatic Environment): A Water Scanning Device for Microplastics
}

\author{
Roxanne Joy D. Dimaano ${ }^{1}$, Alexis C. Albo ${ }^{2}$, Ayesha Xillaine Zaira M. Adion ${ }^{3}$, John Kenneth H. Brucal ${ }^{4}$ \\ ${ }^{1}$ MIMAROPA Regional Science High School, Philippines, dimaanoroxanne@yahoo.com \\ ${ }^{2}$ MIMAROPA Regional Science High School, Philippines, alexis.albo@ deped.gov.ph \\ ${ }^{3}$ MIMAROPA Regional Science High School, Philippines, the_ayeshaadion@yahoo.com \\ ${ }^{4}$ MIMAROPA Regional Science High School, Philippines, brucaljk@ gmail.com
}

\begin{abstract}
Microplastics particles have become an important ecological problem due to a huge amount of plastics debris that ends up in the sea. It is expected that the number of affected marine species will rise as research on this topic increases. Deep learning shows a potential for solving complex problems without the need for a physical understanding of the underlying system, and hence offers an elegant solution. The application of convolutional neural networks for the identification of microparticles in different aquatic environment was demonstrated. The measurements were carried out in real-time using a Raspberry Pi, a digital microscopic camera, and neural network computation, hence demonstrating a portable and low-cost environmental aquatic sensor. Phyton programming language was used to encode the input in the raspberry pi which serve as the brain of the device. The network model is trained using 1000 datasets where $70 \%$ was designed for training and validation, and $30 \%$ was for testing. The deep learning approach produced a good performance with $97.65 \%-88.32 \%-84.00 \%$ trainingvalidation-testing accuracy for the Convolutional Neural Network model. The actual field tests conducted, showed a high percentage accuracy (90\%) on different aquatic environment when compared with laboratory tests using Ultraviolet Visible Spectroscopy. The average discharging time was 1.18 hour which denotes that the scanning device can be used for a long period of time. The program and platform of the scanning device were functional. It can be concluded that the water sensing device is a great help in keeping our water clean and safe.
\end{abstract}

Key words: Convolutional Neural Network, Image Processing, Microplastics, Raspberry Pi

\section{INTRODUCTION}

One of the emerging ecologic problem today is the microplastic particles. Due to the huge amount of plastic debris that ends up in the sea, the expected number of affected marine species will increase as the research on this topic increases. Minimum estimates on the number of marine plastics in the world's oceans are currently placed at 5.25 trillion pieces [1]. This increasing number of plastics in the marine environment through the years could also mean an increase in interactions with different marine species. Overlap within the distribution of marine debris, including marine plastics, and therefore the foraging habitats of marine species will result as marine plastics become more ubiquitous. Previous studies have implicated the Philippines as one of the highest contributors of plastics to the marine environment [2]. The country is claimed to contribute 0.28 0.75 million metric tons of marine plastic per year.

Microplastics, commonly defined as synthetic polymers with a size below $5 \mathrm{~mm}$, which are found in seawater samples from all over the globe, are making headlines as emerging, widespread pollutants (Pham et al., 2014). The plastic pollution of our oceans has proven to be of societal, environmental and economic concern and is included as one of the descriptors for good environmental status (GES) in the marine strategy framework directive (MFDS) [3].

An additional impact is that the ingestion of microplastics by marine species, and thus microplastics enter into the organic phenomenon with unpredictable effects on humans. In addition to the exploration of their presence in fishes, researchers are studying the presence of microplastics in coastal areas. The expansion of protected areas for marine biodiversity and existing policies and treaties that encourage responsible use of plastics are still insufficient to combat the adverse effects of accelerating plastics specifically microplastics floating on the ocean.

The presence of microplastics in the environment is the result of two introduction pathways: as primary microplastics in the form of $e . g$ virgin plastic pellets and powders, by use and disposal of microbeads in cosmetic and cleaning products and as secondary microplastics by the fragmentation of litter. Plastic undergoes mechanical or UVlight induced degradation and is reduced in size. 
Monitoring such particles is essential to help determine origin and distribution, whilst also providing data for mitigating the effects of plastic pollution. Since microplastics can reach the marine floor, having been found in sediments at depths down to $5000 \mathrm{~m}$, and in deposit feeders that ingest sediment, it is therefore important to monitor plastics before they reach the marine floor. Such a sensing device must have the capability to identify the different types of microplastics and natural occurring particles, such as sand, in order for accurate monitoring.

However, a significant problem in monitoring such a global problem is the lack of a reliable portable and low-cost method for characterization of the pollution particles. In this study, the researchers aim to facilitate and accelerate the reliable and clear detection of microplastics in environmental samples. To accomplish this, this study presents an automated analysis approach using image processing.

\section{RELATED WORKS}

Manta nets can be used to collect plastic particulate matter from the marine environment of sizes down to $333 \mu \mathrm{m}$, with additional laboratory sieving used to separate out smaller micro particles [4], and material characterization carried out subsequently using a range of spectroscopic and imaging techniques [5]. Such collection and characterization methods are extremely time-consuming and expensive, and hence alternative methods are required.

A holographic technique that involves analyzing the scattered light from particles that had shown the potential for the characterization of particle contaminants in water was proposed [6]. Although particle monitoring from observation of the scattered light has been demonstrated, such methods require simulations and lack the flexibility for identification of non-spherical particles which is more prevalent on microplastics.

Quantitative measurements are important for risk assessment and monitoring purposes. They are also important in allowing temporal and spatial comparison of pollutants. At present, microplastic surveys require visual analysis, often performed using a microscope. This procedure is time consuming and investigations show that results differ between researchers. A faster and more objective method of analysis, suitable for environmental samples, would therefore be beneficial in future microplastic studies. An increasing number of publications are combining microscopy with spectral analysis of identified particles to avoid misidentification. Often Raman or Fourier transform infrared (FT-IR) spectroscopy is used in combination with visual identification in a microscope. These techniques require individual particle analysis, meaning that the suspected plastic particle has to be visually identified as plastic, or suspected plastic, and then tested spectroscopically. It has, however, been shown that for plastic particles and fragments the particles are likely underestimated [7].
Current microplastic research suffers from insufficient reliable data on concentrations of microplastics in several aquatic environment and on the composition of involved polymers because standard operation protocols (SOP) for microplastic sampling and detection are not available [8].

In recent years due to its state-of-the art performance in many research domains, deep learning has attracted attention of academic community. The practice of the Deep Learning approaches for object classification have exhibited a performance in complex tasks like never before. Obviously, the problem of microplastics classification could be solved with a Deep Learning approach but the lack of thousands of labeled samples hinders the training process.

\section{METHODOLOGY}

The development of a water scanning device for microplastics involves the following: the embedded system hardware has to be selected and configured. Then operating system is installed and configured and finally, actual testing of the device is conducted.

\section{A. Hardware Development}

Shown in figure 1 is the system mechanism of the scanning device (ANTIPARA). Using a USB Digital Microscopic Camera as the scanner, the proposed system can monitor and detect presence of microplastics in water. The classification of microplastic was done by the brain of the system Raspberry Pi 3 model B+ using Convolutional Neural Network as its algorithm. As the camera scans underwater, live video of the process is displayed on the LCD.

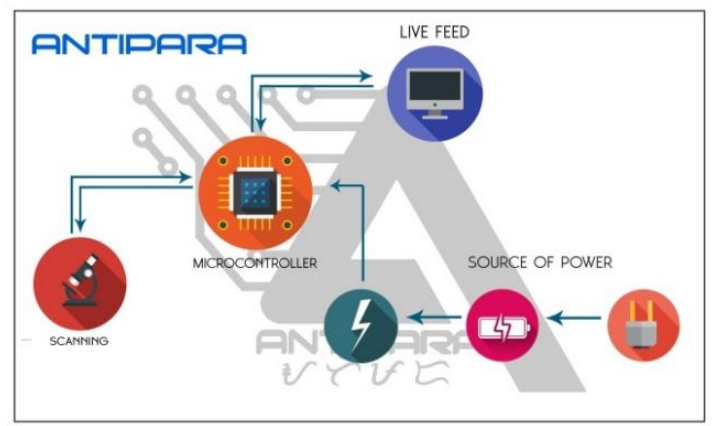

Figure 1: ANTIPARA System Mechanism

The platform of the device is designed to assure the functionality and portability of device. The external cover was made from black box made out from Polypropylene which has high heat resistance to protect the electronics. All the electronics were contained in the black box. The dimension of the device were $20 \mathrm{~cm}(\mathrm{~L}) \times 7 \mathrm{~cm}(\mathrm{H}) \times 16 \mathrm{~cm}$ (W).

\section{B. Development of the Microplastics Detection System}

In general, as shown in Figure 2, any digital image processing algorithm consists of three stages: input, 
processor and output. In the input stage image is captured by a camera. The pixel image of the input change will then focus on a particular system that gives its output as processed image.

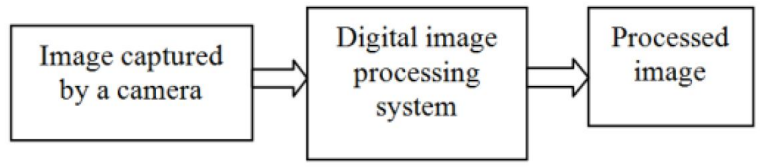

Figure 2: General Block Diagram of Image Processing

A Convolutional Neural Network (CNN) was used, which is a type of neural network designed mainly for image processing, with a probability output. The amount of knowledge available may be a limitation in deep learning and findings its right distribution may be a challenge for improving accuracy for any specific application. CNN consists of multiple layer because it also belongs to the Deep Neural Network [9]. Its functionality is based on the artificial neural network [10]. The CNN model with two layers of 2D convolutional (CONV) layers and an output layer of fullyconnected neural network was used in this study.

For this approach, the dataset is divided into $70 \%$ for the training and 30\% for the testing. The total number of datasets are 1000, which are consisted by 700 datasets for training and 300 for testing. The microscopic images of the microplastics are manually collected using a digital microscope with 1000x magnifier.

The input layer takes an input image with 28 rows, 28 columns, and three channels (RGB) for depth. The researchers then learn 20 convolution filters, each of which are $5 \times 5$. The CONV layer is followed by ReLU activation followed by $2 \times 2$ max pooling in both $\mathrm{x}$ and $\mathrm{y}$ direction with a stride of two. The next block of the architecture follows the same pattern, this time learning $505 \times 5$ filters. It is common to see the number of CONV layers increase in deeper layers of the network as the actual spatial input dimension decrease. The researcher then has two FC layers. The first FC contains 500 hidden nodes followed by a ReLU activation. The final FC layer controls the number of output class labels. Finally, the researchers applied a softmax activation to obtain the class probabilities. The $\mathrm{CNN}$ model and its parameters are listed in Table 1.

Table 1: CNN Model Parameters

\begin{tabular}{|l|l|}
\hline \multicolumn{1}{|c|}{ CNN Model } & \multicolumn{1}{c|}{ Parameters } \\
\hline $\begin{array}{l}\text { Input Layer } \\
\text { (2D Convolutional Layer) }\end{array}$ & $\begin{array}{l}\text { 20 filters } \\
5 \times 5 \text { convolution size }\end{array}$ \\
\hline Activation & Rectified Linear unit \\
\hline 2D Max Pooling Layer & 2x2 pool size \\
\hline 2D Convolutional Layer & $\begin{array}{l}20 \text { filters } \\
5 \times 5 \text { convolution size }\end{array}$ \\
\hline Activation & Rectified Linear unit \\
\hline 2D Max Pooling Layer & $2 \times 2$ pool size \\
\hline $\begin{array}{l}\text { Core Layer } \\
\text { (Fully-connected Neural } \\
\text { Network Layer) }\end{array}$ & 500 nodes \\
\hline
\end{tabular}

\begin{tabular}{|l|l|}
\hline Activation & Rectified Linear unit \\
\hline $\begin{array}{l}\text { Fully-connected Neural } \\
\text { Network Layer }\end{array}$ & 2 filters (output) \\
\hline Activation & Softmax \\
\hline
\end{tabular}

\section{Data Analysis and Testing}

Microplastics are plastic particles that are smaller than 5.0 $\mathrm{mm}$ in size. The researchers prepared 10 setups of the sample with $5 \mathrm{~mm}, 4 \mathrm{~mm}, 3 \mathrm{~mm}, 2 \mathrm{~mm}, 1 \mathrm{~mm}$ and less than $1 \mathrm{~mm}$ microplastics were present. The water samples were from the tap water and sea water. Samples were captured and analyzed at science laboratory. Figure 3a shows microplastic samples from the plastic debris while figure $3 b$ shows samples from plastics wrappers.

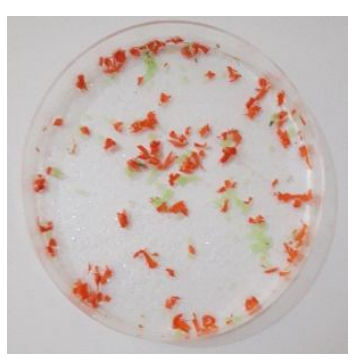

(a)

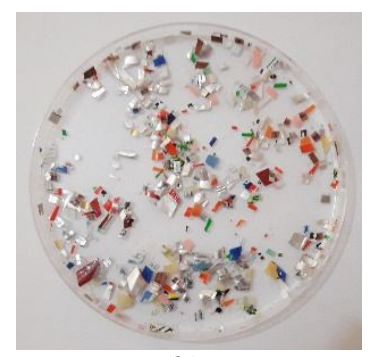

(b)
Figure 3: Examples of Microplastics (a) debris from PVC pipes and (b) plastic wrappers

In deep learning applications, data augmentation is simplest and most widely used method to increase the number of images in the data set, to further the model's performance and to reduce overfitting in the image data [10]. This method aims to help the network learn the feature better. This can be achieved by random transformations such as rotation, width shift, height shift, rescale, shear, zoom, and horizontal flip. Data augmentation also prevent overfitting and helps the learning model to have prediction bias.

The study used the Keras library to pre-process image. Table 2 lists the parameters used in the data augmentation process. Data augmentation process is used for the microplastics dataset.

Table 2: Data Augmentation Parameter

\begin{tabular}{|c|c|}
\hline Parameter & Value \\
\hline Rotation Range & 30 \\
\hline Width Shift & 0.1 \\
\hline Height Shift & 0.1 \\
\hline Shear Range & 0.2 \\
\hline Zoom Range & 0.2 \\
\hline Horizontal Flip & True \\
\hline Fill Mode & Nearest \\
\hline
\end{tabular}

The researchers used ANTIPARA to identify the presence of microplastics in each prepared sample. For the ten setups, there were five trials conducted for every setup. The 
researchers computed for the accuracy (correct analysis/total number of trials) of the device in identifying microplastics.

ANTIPARA was used in an actual aquatic environment. The researchers used the device in different water environment like seawater, river water and water creeks within the province of Oriental Mindoro, Philippines. In order to identify the accuracy of the analysis of the device, the researchers collected the water samples and subjected it to actual laboratory testing for microplastics. Figure 4 shows the testing done using a UV Visible Spectroscopy machine. A total of ten testing were done.

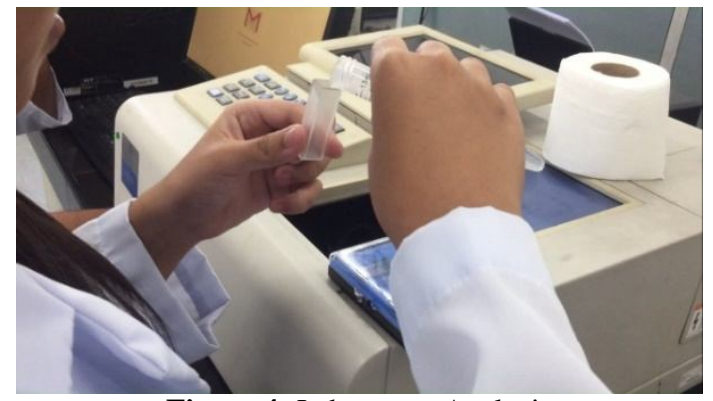

Figure 4: Laboratory Analysis

\section{RESULTS AND DISCUSSION}

Table 3 shows the functionality of the controls and programs of the device. In terms of the functionality of the controls of the scanning device, it can be seen from the table that the controls are fully functional. This means that the connections made through the device are correct and functional.

Table 3: Functionality of the controls of the scanning device

\begin{tabular}{ll}
\hline Command & Activity \\
\hline On & Responding \\
Off & Responding \\
Reboot & Responding \\
Run & Responding \\
Shutdown & Responding \\
\hline
\end{tabular}

The CNN model used a total of 400 training and 300 validation images. The learning model was simulated for 75 seconds with 25 epochs. The model results shown in table 4 revealed that the training accuracy is $97.65 \%$ with a loss function of 0.0798 , while the validation accuracy is $88.32 \%$ with a loss function of 0.4234 .

Table 4: Accuracy Performance of CNN Model

\begin{tabular}{lc}
\hline Results & Value \\
\hline Training Accuracy & $97.65 \%$ \\
Validation Accuracy & $88.32 \%$ \\
Training Loss Function & 0.0798 \\
Validation Loss Function & 0.4234 \\
Number of Epochs & 25 \\
Simulation time (seconds) & 75 \\
\hline
\end{tabular}

The trained deep learning model was evaluated in actual set up using separate 50 water samples. Figure 5 shows the accuracy of identifying microplastics by ANTIPARA in different actual setups. It can be gleaned from the figure that there is a good result on the analysis by the device with 84 percentage of accuracy or forty-two (42) correct analysis out of fifty (50) total number of setups.

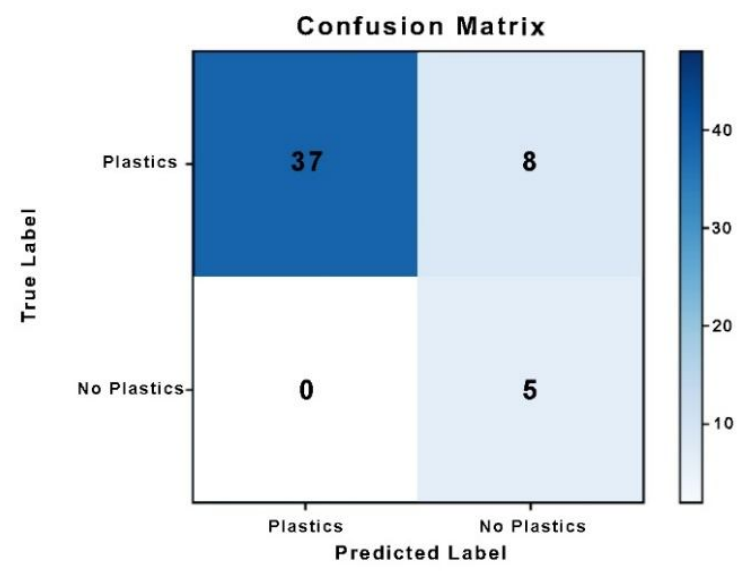

Figure 5: Confusion Matrix

Table 5 shows the comparison between the analysis of the scanning device (ANTIPARA) on different water sample in Oriental Mindoro and the UV-Visible Spectroscopy on identifying the presence of microplastics. It can be gleaned from the table that out of ten (10) water samples, nine (9) were correctly identified by the device ( $90 \%$ accuracy). This means that ANTIPARA provides an accurate and comparable result with UV-Visible Spectroscopy results.

Table 5. Comparison Between ANTIPARA and UV-Visible Spectroscopy (No absorbance means no microplastics present; With absorbances means microplastics are present) on Identifying Presence of Microplastics

\begin{tabular}{|c|c|c|}
\hline Water Sample & $\begin{array}{c}\text { Device } \\
\text { Analysis }\end{array}$ & UV-Vis Result \\
\hline 1. Distilled Water & No Plastic & No absorbance \\
\hline 2. Sea Water $_{1}$ & No Plastic & No absorbance \\
\hline 3. Sea Water 2 & No Plastic & No absorbance \\
\hline 4. Sea Water $_{3}$ & No Plastic & No absorbance \\
\hline 5. River Water $_{1}$ & No Plastic & No absorbance \\
\hline 6. River Water 2 & No Plastic & No absorbance \\
\hline 7. River Water $_{3}$ & No Plastic & No absorbance \\
\hline 8. Lake Water $_{1}$ & Plastic & No absorbance \\
\hline 9. Lake Water $_{2}$ & No Plastic & No absorbance \\
\hline 10. Creek & Plastic & With absorbance \\
\hline
\end{tabular}

Figure 6 shows the analysis of the scanning device on water sample found in a creek on Bansud, Oriental Mindoro. On table 5, two out of ten water samples were revealed to have the presence of microplastics. The other sample is from Naujan Lake. On the other hand, to test the accuracy of the scanning device, all ten samples were subjected to UVVisible Spectroscopy. The UV-Visible Spectroscopy 
machine used a wavelength scan starting from $1000 \mathrm{~nm}$ and ending at $200 \mathrm{~nm}$ with a scan speed of $800 \mathrm{~nm} / \mathrm{min}$ at $1 \mathrm{~nm}$ interval. Figure 7 shows the result of the UV-Visible Spectroscopy of the water sample found on the creek. It can be seen from the figure that the graph contains peak at $233 \mathrm{~nm}$ therefore the water sample contains microplastics. Further, UV-Visible Spectroscopy result of the water sample found at Naujan lake has no peak which means it has no microplastics present on it.

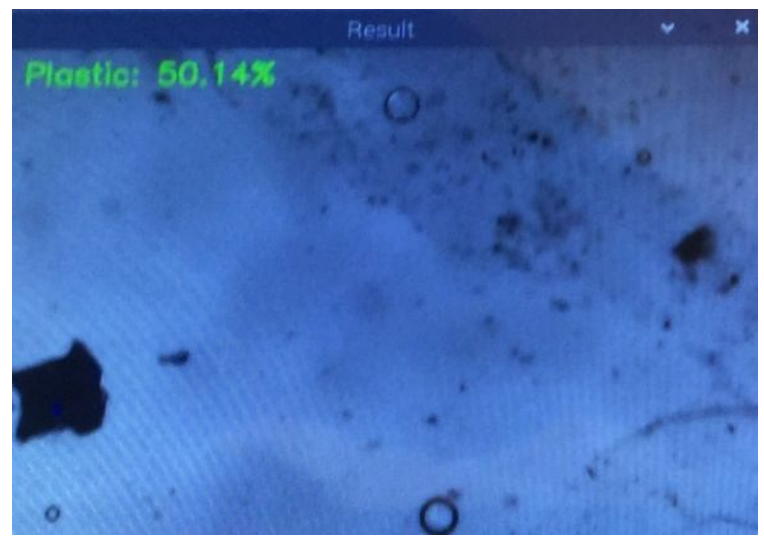

Figure 6: Analysis of ANTIPARA on Creek

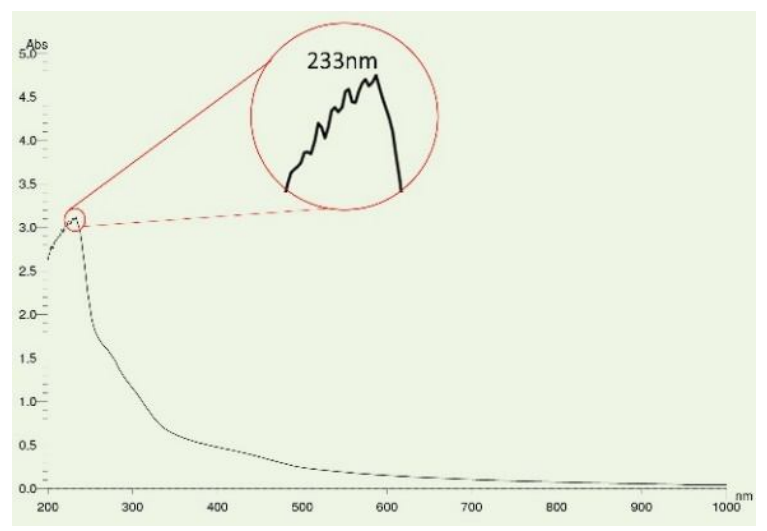

Figure 7: UV-Visible Spectroscopy on Creek

\section{CONCLUSION}

This paper introduced a water scanning device (ANTIPARA) capable of identifying the presence of microplastics using image processing and convolutional neural network. Based from the results gathered from the testing made, it can be concluded that ANTIPARA provides an accurate and efficient analysis of water samples for identifying microplastics. The device was able to successfully identify microplastics in samples such as distilled water, sea water, river water, lake water and tap water. The performance of the device can be credited to the deep learning approach which produced $97.65 \%$ - $88.32 \%-84.00 \%$ training-validationtesting accuracy. In addition, the scanning device can be used for a long period of time. Thus, ANTIPARA, a water scanning device will be a great help in keeping our water clean and safe.

\section{REFERENCES}

1. M. Eriksen, A. Cummins, N. Maximenko, M. Thiel, G. Lattin, S. Wilson, J. Hafner, A. Zellers, and S. Rifman. Plastic Pollution in the South Pacific Subtropical Gyre. Plastics Engineering, vol. 69, no. 5, pp. 38-44, May 2013.

2. J. R. Jambeck, R. Geyer, C. Wilcox, T. R. Siegler, M. Perryman, A. Andrady, R. Narayan, and K. L. Law, "Plastic waste inputs from land into the ocean" Science, vol. 347, no. 6223, pp. 768-771, Feb. 2015.

3. Olenin, S., F. Alemany, A. C. Cardoso, S. Gollasch, P. Goulletquer, M. Lehtiniemi, T. McCollin et al. "Marine strategy framework directive." Task Group 2 (2010).

4. C. J. Moore. Synthetic polymers in the marine environment: A rapidly increasing, long-term threat. Environmental Research, vol. 108, no. 2, pp. 131-139, Oct. 2008.

5. J. Gasperi, R. Dris, T. Bonin, V. Rocher, and B. Tassin. Assessment of floating plastic debris in surface water along the Seine River. Environmental Pollution, vol. 195, pp. 163-166, Dec. 2014.

6. L. A. Philips, D. B. Ruffner, F. C. Cheong, J. M. Blusewicz, P. Kasimbeg, B. Waisi, J. R. McCutcheon, and D. G. Grier. Holographic characterization of contaminants in water: Differentiation of suspended particles in heterogeneous dispersions. Water Research, vol. 122, pp. 431-439, Oct. 2017.

7. Y. K. Song, S. H. Hong, M. Jang, G. M. Han, M. Rani, J. Lee, and W. J. Shim. A comparison of microscopic and spectroscopic identification methods for analysis of microplastics in environmental samples. Marine Pollution Bulletin, vol. 93, no. 1-2, pp. 202-209, Apr. 2015.

8. V. Hidalgo-Ruz, L. Gutow, R. C. Thompson, and M. Thiel. Microplastics in the Marine Environment: A Review of the Methods Used for Identification and Quantification. Environmental Science \& Technology, vol. 46, no. 6, pp. 3060-3075, Mar. 2012.

9. Bharat, Vinod \& Malik, Dr. Study of Detection of Various types of Cancers by using Deep Learning: A Survey. International Journal of Advanced Trends in Computer Science and Engineering. Vol. 8 no 4, pp. 1228-1233, June 2019. https://doi.org/10.30534/ijatcse/2019/31842019

10. Jay Robert B. Del Rosario. Development of Face Recognition System Using Deep Convolutional Neural Networks in a Multi View Vision Environment, International Journal of Advanced Trends in Computer Science and Engineering, Vol 8 no 3, pp. 369-374, June 2019. https://doi.org/10.30534/ijatcse/2019/06832019

11. A. Krizhevsky, I. Sutskever, and G. E. Hinton. ImageNet classification with deep convolutional neural networks. Communications of the ACM, vol. 60, no. 6, pp. 84-90, May 2017. 